\title{
GENERIC HOMEOMORPHISMS HAVE THE PSEUDO-ORBIT TRACING PROPERTY
}

\author{
KENZI ODANI \\ (Communicated by Kenneth R. Meyer)
}

\begin{abstract}
Let $M(\operatorname{dim} M \leq 3)$ be a compact manifold. Then a generic $f \in$ Homeo $(M)$ satisfies the following: $f$ has the pseudo-orbit tracing property; $f$ is $C^{0}$ tolerance stable; and $f$ is not topologically stable.
\end{abstract}

\section{INTRODUCTION}

Let $M$ be a compact differentiable manifold with the metric $d$ induced by a Riemannian structure. $d$. We denote by $\operatorname{Homeo}(M)$ the space of all homeomorphisms on $M$ with the $C^{0}$ topology, i.e. the topology induced by the following metric.

$$
d(f, g)=\max _{x \in M} d(f(x), g(x)) .
$$

The $C^{0}$ topology depends on neither the differentiable structure nor the Riemannian structure.

A subset in a topological space is called residual if it includes a countable intersection of open and dense subsets. A topological space is called a Baire space if every residual set is dense in it. In particular, every complete metric space is a Baire space. For example, $\operatorname{Homeo}(M)$ is a Baire space because the metric $\tilde{d}$ below, which induces the $C^{0}$ topology, makes $\operatorname{Homeo}(M)$ a complete metric space.

$$
\tilde{d}(f, g)=d(f, g)+d\left(f^{-1}, g^{-1}\right) .
$$

Moreover, the $C^{0}$ closure of all diffeomorphisms, denoted $\operatorname{ClDiff}(M)$, is also a Baire space.

Let $P$ be a property for homeomorphisms. We say that generic homeomorphisms in $\operatorname{Homeo}(M)$ (resp. $\operatorname{ClDiff}(M))$ satisfy $P$ if the set of homeomorphisms satisfying $P$ is residual in $\operatorname{Homeo}(M)$ (resp. $\operatorname{ClDiff}(M))$. There are some results about generic homeomorphisms, for exampic [5, 12], and the following theorem.

Received by the editors May 30, 1989.

1980 Mathematics Subject Classification (1985 Revision). Primary 58F10; Secondary 54H20.

Key words and phrases. Generic homeomorphisms, POTP, tolerance stability, topological stability. 
Theorem ([13]). If $M=S^{1}$, then a generic $f \in \operatorname{Homeo}(M)$ satisfies the following:

(1) $f$ has the pseudo-orbit tracing property;

(2) $f$ is $C^{0}$ tolerance stable; and

(3) $f$ is not topologically stable.

In $\S 3$, we extend the above theorem to the case when $\operatorname{dim} M \leq 3$. The main tool is Shub's density theorem [6].

\section{Definitions}

After this, $f$ and $g$ denote elements of $\operatorname{Homeo}(M)$.

(1) A sequence $\left\{x_{i}\right\}_{i \in \mathbf{Z}}$ is a $\delta$-pseudo-orbit of $f$ if $d\left(f\left(x_{i}\right), x_{i+1}\right) \leq \delta$ for every $i \in \mathbf{Z}$.

(2) A sequence $\left\{x_{i}\right\}_{i \in \mathbf{Z}}$ is $\epsilon$-traced by the $f$-orbit through $x \in M$ if $d\left(f^{i}(x), x_{i}\right) \leq \epsilon$ for every $i \in \mathbf{Z}$.

(3) A sequence $\left\{x_{i}\right\}_{i \in \mathbf{Z}}$ is $\epsilon$-set-traced by the $f$-orbit through $x \in M$ if $\bar{d}\left(\mathrm{Cl}\left\{f^{i}(x): i \in \mathbf{Z}\right\}, \mathrm{Cl}\left\{x_{i}: i \in \mathbf{Z}\right\}\right) \leq \epsilon$.

Here we denote by $\operatorname{Cl}\{*\}$ the closure and by $\bar{d}$ the Hausdoff metric with respect to $d$.

(4) $f$ is strongly $C^{0}$ tolerance stable if, for every $\epsilon>0$, there exists $\delta>0$ such that, for every $g \in V_{\delta}(f)$, every $f$-orbit is $\epsilon$-traced by some $g$ orbit and every $g$-orbit is $\epsilon$-traced by some $f$-orbit.

Here we denote by $V_{\delta}(f)$ the $\delta$-neighborhood of $f$ in $\operatorname{Homeo}(M)$.

(5) $f$ is $C^{0}$ tolerance stable if, for every $\epsilon>0$; there exists $\delta>0$ such that, for every $g \in V_{\delta}(f)$, every $f$-orbit is $\epsilon$-set-traced by some $g$-orbit and every $g$-orbit is $\epsilon$-set-traced by some $f$-orbit.

(6) $f$ has the pseudo-orbit tracing property (abbr. POTP) if, for every $\epsilon>0$, there exists $\delta>0$ such that every $\delta$-pseudo-orbit of $f$ is $\epsilon$ traced by some $f$-orbit.

(7) $f$ is lower semi-conjugate to $g$ under $\varphi$ if there exists a continuous surjection $\varphi: M \rightarrow M$ satisfying $f \varphi=\varphi g$.

(8) $f$ is topologically stable if, for every $\epsilon>0$, there exists $\delta>0$ such that, for every $g \in V_{\delta}(f), f$ is lower semi-conjugate to $g$ under $\varphi$ satisfying $d\left(\varphi, 1_{M}\right) \leq \epsilon$.

By the definition, the topological stability implies the strong $C^{0}$ tolerance stability.

Proposition 1. Let $f$ be strongly $C^{0}$ tolerance stable. Then:

(1) $f$ is $C^{0}$ tolerance stable; and

(2) $f$ has the POTP.

Proof.

(1) It is immediate by the fact that the $\epsilon$-traceability implies the $\epsilon$-settraceability. 
(2) The $m$-dimensional cases $(m \geq 2)$ are proved by modification of the proof of [10, Theorem 11]. The 1-dimensional case is also valid, see Remark 2 below.

Remark 2. There is the necessary and sufficient condition that $f \in \operatorname{Homeo}\left(S^{1}\right)$ has the POTP, see [13]. It is also equivalent to the strong $C^{0}$ tolerance stability, see [4].

\section{MAIN THEOREM}

Theorem A. A generic $f \in \operatorname{CIDiff}(M)$ satisfies the following:

(0) $f$ is strongly $C^{0}$ tolerance stable;

(1) $f$ has the pseudo-orbit tracing property;

(2) $f$ is $C^{0}$ tolerance stable; and

(3) $f$ is not topologically stable.

By the above theorem, for every compact differentiable manifold $M$, we can see the existence of the homeomorphisms which have the POTP but are not topologically stable.

By [2] or [11], if $\operatorname{dim} M \leq 3$, then $\operatorname{ClDiff}(M)=\operatorname{Homeo}(M)$. Therefore we obtain the following theorem as a corollary.

Theorem B. If $\operatorname{dim} M \leq 3$, then a generic $f \in \operatorname{Homeo}(M)$ satisfies the properties (0)-(3) in Theorem A.

\section{Proof of Theorem A}

The combination of Shub's density theorem [6] (or [7]) and Nitecki's topological stability theorem [3] implies the following lemma.

Lemma 3. The set of all topologically stable homeomorphisms is dense in $\operatorname{ClDiff}(M)$.

To prove Theorem A, we introduce some subsets in $\operatorname{ClDiff}(M)$ as follows. $T(M)=\left\{f: f\right.$ is strongly $C^{0}$ tolerance stable $\}$

$T_{\epsilon}(M)=\left\{f:\right.$ there exists $\delta>0$ such that, for every $g \in V_{\delta}(f)$, every $f$-orbit is $\epsilon$-traced by some $g$-orbit and every $g$-orbit is $\epsilon$-traced by some $f$-orbit $\}$.

Lemma 4. For every $\epsilon>0, T_{\epsilon}(M)$ includes an open and dense subset in $\operatorname{ClDiff}(M)$.

Proof. By Lemma 3, $T(M)$ is dense in $\operatorname{ClDiff}(M)$. Therefore it is sufficient to show that every $h \in T(M)$ is an interior point of $T_{\epsilon}(M)$ for every $\epsilon>0$.

Let us take every $h \in T(M)$ and $\epsilon>0$. Then there exists $\delta>0$ such that for every $k \in V_{2 \delta}(h)$, every $h$-orbit is $\epsilon / 2$-traced by some $k$-orbit and every $k$-orbit is $\epsilon / 2$-traced by some $h$-orbit. If $f \in V_{\delta}(h)$ and $g \in V_{\delta}(f)$, then $f$, $g \in V_{2 \delta}(h)$. Therefore, if $f \in V_{\delta}(h)$, then $f \in T_{\epsilon}(M)$. 
By Lemma 4 and the equality below, Theorem A (0) is proved.

$$
T(M)=\bigcap_{n \geq 1} T_{1 / n}(M) .
$$

Moreover, by Proposition 1, (1) and (2) are valid.

By Shub's density theorem, a topologically stable $f \in \operatorname{ClDiff}(M)$ has finite chain components, see [1]. Therefore, by Lemma 5 below, Theorem A (3) is proved. We can prove Lemma 5 by the same method of the proof of [5, Theorem $1(\mathrm{~h})]$.

Lemma 5. A generic $f \in \operatorname{ClDiff}(M)$ has infinite chain components.

\section{ACKNOWLEDGMENT}

The author wishes to thank Professor Kenichi Shiraiwa for criticism and encouragement to form the paper.

\section{REFERENCES}

1. M. Hurley, Consequences of topological stability, J. Differential Equations 54 (1984), 60-72.

2. J. Munkres, Obstructions to the smoothing of piecewise-differentiable homeomorphisms, Ann. Math. 72 (1960), 521-554.

3. Z. Nitecki, On semi-stability for diffeomorphisms, Invent. Math. 14 (1971), 83-122.

4. K. Odani, Generic homeomorphisms have the POTP, in Bifurcation Phenomena in Nonlinear Systems and Theory of Dynamical Systems (H. Kawakami, ed.), World Scientific (to appear).

5. J. Palis, C. Pugh, M. Shub and D. Sullivan, Genericity theorems in topological dynamics, Springer Lecture Notes, vol. 468, 1975, pp. 241-250.

6. M. Shub, Structurally stable diffeomorphims are dense, Bull. Amer. Math. Soc. 78 (1972), 817-818.

7. M. Shub and D. Sullivan, Homology theory and dynamical systems, Topology 14 (1975), 109-132.

8. F. Takens, On Zeeman's tolerance stability conjecture, Springer Lecture Notes, vol. 197, 1971, pp. 209-219.

9. __ Tolerance stability, Springer Lecture Notes, vol. 468, 1975, pp. 293-304.

10. P. Walters, On the pseudo-orbit tracing property and its relationship to stability, Springer Lecture Notes, vol. 668, 1979, pp. 231-224.

11. J. H. C. Whitehead, Manifolds with transverse fields in Euclidean space, Ann. Math. 73 (1961), 154-212.

12. K. Yano, A remark on the topological entropy of homeomorphisms, Invent. Math. 59 (1980), 215-220.

13. __ Generic homeomorphisms of $S^{1}$ have the pseudo-orbit tracing property, J. Fac. Sci. Univ. Tokyo Sect. IA Math. 34 (1987), 51-55. JAPAN

Department of Mathematics, Faculty of Science, Nagoya University, Nagoya 464-01, 